Feature Selection with Neural Networks

Philippe LERAY and Patrick GALLINARI

\author{
LIP6 - Pôle IA \\ Université Paris 6 - boite 169 \\ 4, Place Jussieu \\ 75252 Paris cedex 05 \\ France \\ \{Philippe.Leray, Patrick.Gallinari\}@lip6.fr
}




\title{
Feature Selection with Neural Networks
}

\author{
Philippe LERAY and Patrick GALLINARI
}

\begin{abstract}
Features gathered from the observation of a phenomenon are not all equally informative: some of them may be noisy, correlated or irrelevant. Feature selection aims at selecting a feature set that is relevant for a given task. This problem is complex and remains an important issue in many domains. In the field of neural networks, feature selection has been studied for the last ten years and classical as well as original methods have been employed. This paper is a review of neural network approaches to feature selection. We first briefly introduce baseline statistical methods used in regression and classification. We then describe families of methods which have been developed specifically for neural networks. Representative methods are then compared on different test problems.
\end{abstract}

Keywords

Feature Selection, Subset selection, Variable Sensitivity, Sequential Search 


\title{
Sélection de Variables et Réseaux de Neurones
}

\author{
Philippe LERAY et Patrick GALLINARI
}

\begin{abstract}
Résumé
Les données collectées lors de l'observation d'un phénomène ou mesurées sur un système physique ne sont pas toutes aussi informatives: certaines variables peuvent correspondre à du bruit, être peu significatives, corrélées ou non pertinentes pour la tâche à réaliser. La sélection de variables est donc un problème complexe et fait l'objet de recherches dans de nombreuses disciplines. Dans le domaine des réseaux de neurones, la sélection de variables est étudiée depuis une dizaine d'années et un certain nombre de méthodes, plus ou moins proches des méthodes classiques, ont émergé. Ce rapport est une revue des approches connexionnistes pour la sélection de variables. Nous présentons tout d'abord les différents éléments constituant une méthode de sélection de variables. Nous évoquons ensuite brièvement les méthodes statistiques utilisées en régression et en classification. Nous passons enfin en revue les principales méthodes développées spécialement pour les réseaux de neurones. Plusieurs méthodes représentatives de sélection de variables sont alors comparées sur différents problèmes.
\end{abstract}

Mots Clés

Sélection de Variables, Sélection de Caractéristiques, Pertinence d'une Variable, Recherche Séquentielle 


\title{
Feature Selection with Neural Networks
}

\author{
Philippe LERAY and Patrick GALLINARI
}

\section{Introduction}

Learning systems primary source of information is data. For numerical systems like Neural Networks (NNs), data are usually represented as vectors in a subspace of $R^{k}$ whose components - or features - may correspond for example to measurements performed on a physical system or to information gathered from the observation of a phenomenon. Usually all features are not equally informative: some of them may be noisy, meaningless, correlated or irrelevant for the task. Feature selection aims at selecting a subset of the features which is relevant for a given problem. It is most often an important issue: the amount of data to gather or process may be reduced, training may be easier, better estimates will be obtained when using relevant features in the case of small data sets, more sophisticated processing methods may be used on smaller dimensional spaces than on the original measure space, performances may increase when non relevant information do not interfere, etc.

Feature selection has been the subject of intensive researches in statistics and in application domains like pattern recognition, process identification, time series modelling or econometrics. It has recently began to be investigated in the machine learning community which has developed its own methods. Whatever the domain is, feature selection remains a difficult problem. Most of the time this is a non monotonous problem, i.e. the best subset of $p$ variables does not always contain the best subset of $q$ variables $(q<p)$. Also, the best subset of variables depends on the model which will be further used to process the data - usually, the two steps are treated sequentially. Most methods for variable selection rely on heuristics which perform a limited exploration on the whole set of variable combinations.

In the field of NNs, feature selection has been studied for the last ten years and classical as well as original methods have been employed. We discuss here the problem of feature selection specifically for NNs and review original methods 
which have been developed in this field. We will certainly not be exhaustive since the literature in the domain is already important, but the main ideas which have been proposed are described.

We describe in sections 2 and 3 the basic ingredients of feature selection methods and the notations. We then briefly present, in section 4, statistical methods used in regression and classification. They will be used as baseline techniques. We describe, in section 5, families of methods which have been developed specifically for neural networks and may be easily implemented either for regression or classification tasks. Representative methods are then compared on different test problems in section 6 .

\section{Basic ingredients of feature selection methods.}

A feature selection technique typically requires the following ingredients:

- a feature evaluation criterion to compare variable subsets, it will be used to select one of these subsets,

- a search procedure, to explore a (sub)space of possible variable combinations,

- a stop criterion or a model selection strategy.

\subsubsection{Feature evaluation}

Depending on the task (e.g. prediction or classification) and on the model (linear, logistic, neural networks...), several evaluation criteria, based either on statistical grounds or heuristics, have been proposed for measuring the importance of a variable subset. For classification, classical criteria use probabilistic distances or entropy measures, often replaced in practice by simple interclass distance measures. For regression, classical candidates are prediction error measures. A survey of classical statistical methods may be found in (Thomson 1978) for regression and (McLachlan 1992) for classification.

Some methods rely only on the data for computing relevant variables and do not take into consideration the model which will then be used for processing these data after the selection step. They may rely on hypothesis about the data distribution (parametric methods) or not (non parametric methods). Other 
methods take into account simultaneously the model and the data - this is usually the case for $\mathrm{NN}$ variable selection.

\subsubsection{Search}

In general, since evaluation criteria are non monotonous, comparison of feature subsets amounts to a combinatorial problem (there are $2^{k}-1$ possible subsets for $k$ variables), which rapidly becomes computationally unfeasible, even for moderate input size. Branch and Bound exploration (Narendra and Fukunaga 1977) allows to reduce the search for monotonous criteria, however the complexity of these procedures is still prohibitive in most cases. Due to these limitations, most algorithms are based upon heuristic performance measures for the evaluation and sub-optimal search. Most sub-optimal search methods follow one of the following sequential search techniques (see e.g. Kittler, 1986) :

- start with an empty set of variables and add variables to the already selected variable set (forward methods)

- start with the full set of variables and eliminate variables from the selected variable set (backward methods)

- start with an empty set and alternate forward and backward steps (stepwise methods). The Plus $l$ - Take away $r$ algorithm is a generalisation of the basic stepwise method which alternates $l$ forward selections and $r$ backward deletions.

\subsubsection{Subset selection - Stopping criterion}

Let be given a feature subset evaluation criterion and a search procedure. Several methods examine all the subsets provided by the search (e.g. $2^{k}-1$ for an exhaustive search or $k$ for a simple backward search) and select the most relevant according to the evaluation criterion.

When the empirical distribution of the evaluation measure or of related statistics is known, tests may be performed for the (ir)relevance hypothesis of an input variable. Classical sequential selection procedures use a stop criterion: they examine the variables sequentially and stop as soon as a variable is found irrelevant according to a statistical test. For classical parametric methods, distribution characteristics (e.g. estimates of the evaluation measure variance) are easily derived (see sections 4.1 and 4.2). For non parametric or flexible methods 
like NNs, these distributions are more difficult to obtain. Confidence intervals which would allow to perform significance testing might be computed via monte carlo simulations or bootstrapping. This is extremely prohibitive and of no practical use except for very particular cases (e.g. Baxt and White 1996). Hypothesis testing is thus seldom used with these models. Many authors use instead heuristic stop criteria.

A better methodology, whose complexity is still reasonable in most applications, is to compute for the successive variable subsets provided by the search algorithm an estimate of the generalization error (or prediction risk) obtained with this subset. The selected variables will be those giving the best performances. The generalization error estimate may be computed using a validation set or cross-validation or algebraic methods although the latter are not easy to obtain with non linear models. Note that this strategy involves retraining a $\mathrm{NN}$ for each subset.

\section{Notations}

We will denote $(\boldsymbol{x}, \boldsymbol{y}) \in \mathfrak{R}^{k} \times \mathfrak{R}^{g}$ the realization of a random variable pair $(X, Y)$ with probability distribution $P . x_{i}$ will be the $i^{\text {th }}$ component of $x$ and $x^{l}$ the $l^{\text {th }}$ pattern in a given data set $D$ of cardinality $N$. In the following, we will restrict ourselves to one hidden layer NNs, the number of input and output units will be denoted respectively by $k$ and $g$. The transfer function of the network will be denoted $f$. Training will be performed here according to a Mean Squared Error criterion (MSE) although this is not restrictive. We will consider, in the following, selection methods for classification and regression tasks.

\section{Model independent Feature Selection}

We introduce below some methods which perform the selection and the classification or regression steps sequentially, i.e. which do not take into account the classification or regression model during selection. These methods are not $\mathrm{NN}$ oriented and are used here for the experimental comparison with $\mathrm{NN}$ specific selection techniques (section 6). The first two are basic statistical techniques 
aimed respectively at regression and classification. These methods are not well fitted for NNs since the hypothesis they rely on do not correspond to situations where NNs might be useful. However since most NN specific methods are heuristics they should be used for a baseline comparison. The third one has been developed more recently and is a general selection technique which is data hypothesis free and might be used for any system either for regression or classification. It is based on a probabilistic dependence measure between two sets of variables.

\subsection{Feature selection for linear regression}

We will consider only linear regression, but the approach described below may be trivially extended for multiple regression. Let $x_{1}, x_{2}, \ldots x_{k}$ and $y$ be real variables which will be supposed centered. Let us denote:

$$
f_{(p)}(\mathbf{x})=\sum_{i=1}^{p} b_{i} x_{i}
$$

the current approximation of $y$ with $p$ selected variables (the $x_{i}$ are renumbered so that the $\mathrm{p}$ first selected variables correspond to numbers 1 to $p$ ). The residuals $e_{(p)}=f_{(p)}(\boldsymbol{x})-y$ are assumed identically and independently distributed.

Let us denote:

$$
S S T=\sum_{l=1}^{N}\left(y^{l}\right)^{2} \quad S S R_{p}=\sum_{l=1}^{N} f_{(p)}^{2}\left(\mathbf{x}^{l}\right)
$$

For forward selection, the choice of the $p^{\text {th }}$ variable is usually based on $R_{p}{ }^{2}$, the partial correlation coefficient (table 1) between $y$ and regressor $f(p)$, or on an adjusted coefficient ${ }^{1}$. This coefficient represents the proportion of $y$ total variance explained by the regressor $f_{(p)}$. The $p^{\text {th }}$ variable to select is the one for which $f(p)$ maximizes this coefficient. The importance of a new variable is usually measured via a Fisher test (Thompson, 1978) which compares the models with $p-1$ and $p$ variables $(F s(p)$ forward in table 1$)$. Selection is stopped if

${ }^{1}$ The adjusted coefficient $\bar{R}_{p}^{2}=\frac{N * R_{p}^{2}-p}{N-p}$ is often used instead of $R_{p}^{2}$. 
$F s(p)$ forward $<F(1, N-p, \alpha)$ the Fisher statistics with $(1, N-p)$ degrees of freedom for a confidence level of $\alpha$.

\begin{tabular}{|ccc|}
\hline \hline & Choice & Stop \\
\hline \hline Forward & $R_{p}^{2}=\frac{S S R_{p}}{S S T}=\frac{\sum_{1}^{N} f_{(p)}^{2}\left(x^{l}\right)}{\sum_{1}^{N}\left(y^{l}\right)^{2}}$ & $F S(p)_{\text {forward }}=\frac{S S R_{p}-S S R_{p-1}}{\left(S S T-S S R_{p}\right) /(N-p)}$ \\
Backward & $S S R_{p-1}=\sum_{l=1}^{N} f_{(p-1)}\left(x^{l}\right)^{2}$ & $F S(p)_{\text {backward }}=\frac{S S R_{p-1}-S S R_{p}}{\left(S S T-S S R_{p}\right) /(N-p)}$ \\
\hline \hline
\end{tabular}

Table 1: Choice and Stop criteria used with statistical forward and backward methods.

Note that $F_{S}$ could also be used in place of $R_{p}^{2}$ as a choice criterion:

$$
F s(p)_{\text {forward }}=\frac{R_{p}^{2}-R_{p-1}^{2}}{R_{p}^{2} /(N-p)}
$$

When $p$ - 1 variables have already been selected, $R_{p-1}{ }^{2}$ has a constant value in $[0,1]$ and maximizing $F_{S}$ is similar to maximizing $R_{p}{ }^{2}$. Equation (4.1.3) selects variables in the same order as $R_{p}{ }^{2}$ does.

For backward elimination the variable eliminated from the remaining $p$ is the less significant in terms of the Fisher test i.e. it is the one with the smallest value of $S S R_{p-1}$ or equivalently of $F s(p)$ backward (table 1). Selection is stopped if $F s(p)$ backward $>F(1, N-p, \alpha)$.

\subsection{Feature Selection For Classification}

For classification, we shall select the variable subset which allows the best separation of the data. Variable selection is usually performed by considering a class separation criterion for the choice criterion and an associated F-test as stopping criterion. As for regression, forward, backward or stepwise methods may be used.

Data separation is usually computed through an inter-class distance measure (Kittler, 1986). The most frequent discriminating measure is the Wilks lambda (Wilks, 1963) $\Lambda_{s v p}$ defined as follows: 


$$
\Lambda_{S V_{p}}=\frac{|\boldsymbol{W}|}{|\boldsymbol{W}+\boldsymbol{B}|}
$$

where $\boldsymbol{W}$ is the intra-class matrix dispersion corresponding to the selected variable set $S V_{p}, \boldsymbol{B}$ the corresponding inter-class matrix ${ }^{2}$ and $|\boldsymbol{M}|$ the determinant of matrix $\boldsymbol{M}$. The determinant of a covariance matrix being a measure of the volume occupied by the data, $|\boldsymbol{W}|$ measures the mean volume of the different classes and $|\boldsymbol{W}+\boldsymbol{B}|$ the volume of the whole data set. These quantities are computed for the selected variables so that a good discriminating power corresponds to a small value of $\Lambda_{s v}$ : the different classes are represented by compact clusters and are well separated. This criterion is well suited in the case of multinormal distributions with equal covariance for each class, it is meaningless for e.g. multimodal distributions. This is clearly a very restrictive hypothesis. With this measurement the statistic $F_{S}$, defined below, has a $F(g-1, N-g-p+1, \alpha)$ distribution (McLachlan 1992):

$$
F_{S}=\frac{(N-g-p+1)}{(g-1)} \frac{\left(1-\Lambda_{S V_{p}}\right)}{\Lambda_{S V_{p}}}
$$

We can then use the Wilks lambda both for estimating the discriminating power of a variable and for stopping the selection in forward, backward (Habbema and Hermans, 1977) or stepwise methods.

For the comparisons in section 6, we used Stepdisc, a stepwise method based on (4.2.2) with a 95\% confidence level.

\subsection{Mutual Information}

When data are considered as realization of a random process, probabilistic information measures may be used in order to compute the relevance of a set of

2 these two quantities are defined as :

$$
\boldsymbol{W}=\sum_{j=1}^{g} \sum_{\boldsymbol{x}^{l} \in \text { class } j}\left(\boldsymbol{x}^{l}-\mu_{j}\right)^{t}\left(\boldsymbol{x}^{l}-\mu_{j}\right) \quad \boldsymbol{B}=\sum_{j=1}^{g} N_{j}\left(\mu-\mu_{j}\right)^{t}\left(\mu-\mu_{j}\right)
$$

with $g$ the number of classes, $n_{j}$ the number of samples in class $j, \mu_{j}$ the mean of class $j$ and $\mu$ the global mean. 
variables with respect to other variables. Mutual information is such a measure which is defined as:

$$
M I(a, b)=\sum_{a, b} P(a, b) \times \log \left(\frac{P(a, b)}{P(a) P(b)}\right)
$$

where $a$ and $b$ are two variables with probability density $P(a)$ and $P(b)$.

Mutual information is independent from any inversible and differentiable transformation of the variables. It measures the "uncertainty reduction" on $b$ when $a$ is known. It is also known as the Kullbak-Leibler distance between the joint distribution $P(a, b)$ and the marginal distribution product $P(a) * P(b)$.

The method described below does not make use of restrictive assumptions on the data and is therefore more general and attractive than the ones described in sections 4.1 and 4.2, especially when these hypothesis do not correspond to the data processing model, which is usually the case for NNs. It may be used either for regression or discrimination. On the other hand such non parametric methods are computationally intensive. The main practical difficulty here is the estimation of the joint density $P(a, b)$ and of the marginal densities $P(a)$ and $P(b)$. Non parametric density estimation methods are costly in high dimensions and necessitate a large amount of data.

The algorithm presented below uses the Shannon entropy (denoted $H($.$) ) to$ compute the mutual information $M I(a, b)=H(a)+H(b)-H(a, b)$. It is possible to use other entropy measures like quadratic or cubic entropies (Kittler, 1986).

Battiti (1994) proposed to use mutual information with a forward selection algorithm called MIFS (Mutual Information based Feature Selection). $P(a, b)$ is estimated by Fraser algorithm (Fraser and Swinney, 1986), which recursively partitions the space using $\chi^{2}$ tests on the data distribution. This algorithm can only compute the mutual information between two variables. In order to compute the mutual information between $x_{p}$ and the selected variable set $S V_{p-1}$ ( $x_{p}$ does not belong to $S V_{p-1}$ ), Battiti uses simplifying assumptions. Moreover, the number of variables to select is fixed before the selection. This algorithms uses forward search and variable $x_{p}$ is the one which maximises the value :

$$
\operatorname{MI}\left(S V_{p-1} \cup\left\{x_{p}\right\}, \mathbf{y}\right)
$$

where $S V_{p-1}$ is the set of $p-1$ already selected variables. 
Bonnlander and Weigend (1994) use Epanechnikov kernels for density estimation (Härdle, 1990) and a Branch\&Bound (B\&B) algorithm for the search (Narendra and Fukunaga, 1977). B\&B warrants an optimal search if the criterion used is monotonous and it is less computationally intensive than exhaustive search. For the search algorithm, one can also consider the suboptimal floating search techniques proposed by Pudil et al. (1994) which offer a good compromise between the sequential methods simplicity and the relative computational cost of the Branch\&Bound algorithm.

For the comparisons in section 6, we have used Epanechnikov kernels for density estimation in (4.3.2), a forward search, and the selection is stopped when the MI increase falls below a fixed threshold (0.99).

\section{Model dependent feature selection for Neural Networks}

Model dependent feature selection attempts to perform simultaneously the selection and the processing of the data: the feature selection process is part of the training process and features are sought for optimizing a model selection criterion. This "global optimization" looks more attractive than modelindependent selection where the adequacy of the two steps is up to the user. However, since the value of the choice criterion depends on the model parameters, it might be necessary to train the $\mathrm{NN}$ with different sets of variables: some selection procedures alternate between variable selection and retraining of the model parameters. This forbids the use of sophisticated search strategies which would be computationally prohibitive.

Some specificities of NNs should also be taken into consideration when deriving feature selection algorithms:

- NNs are usually non linear models. Since many parametric modelindependent techniques are based on the hypothesis that input-output variables dependency is linear or that input variables redundancy is well measured by linear correlation between these variables, such methods are clearly ill fitted for NNs.

- The search space has usually many local minima, and relevance measures will depend on the minimum the NN will have converged to. These 
measures should be averaged over several runs. For most applications this is prohibitive and has not been considered here.

- Except for (White 1989) who derives results on the weight distribution there is no work in the NN community which might be used for hypothesis testing.

For NN feature selection algorithms, choice criteria are mainly based on heuristic individual feature evaluation functions. Several of them have been proposed in the literature, we have made an attempt to classify them according to their similarity. We will distinguish between:

- zero order methods which use only the network parameter values.

- first order methods which use the first derivatives of network parameters.

- second order methods which use second derivatives of network parameters.

Most feature evaluation criteria only allow to rank variables at a given time, the value of the criterion by itself being non informative. However, we will see that most of these methods work reasonably well.

Feature selection methods with neural networks use mostly backward search although some forward methods have also been proposed (Moody 1994, Goutte 1997). Several methods use individual evaluation of the features for ranking them and do not take into consideration their dependencies or their correlations. This may be problematic for selecting minimal relevant sets of variables. Using the correlation as a simple dependence measure is not enough since NNs capture non linear relationships between variables, on the other hand, measuring non linear dependencies is not trivial. While some authors simply ignore this problem, others propose to select only one variable at a time and to retrain the network with the new selected set before evaluating the relevance of remaining variables. This allows to take into account some of the dependencies the network has discovered among the variables.

More critical is the difficulty for defining a sound stop criterion or model choice. Many methods use very crude techniques for stopping the selection, e.g. a threshold on the choice criterion value, some rank the different subsets using an 
estimation of the generalization error. The latter is the expected error performed on future data and is defined as:

$$
R=\int r(\boldsymbol{x}, \boldsymbol{y}) p(\boldsymbol{x}, \boldsymbol{y}) d \boldsymbol{x} d \boldsymbol{y}
$$

where in our case, $r(\boldsymbol{x}, \boldsymbol{y})$ is the euclidean error between desired and computed outputs. Estimates can be computed using a validation set, cross-validation or algebraic approximations of this risk like the Final Prediction Error, (Akaike 1970). Several estimates have been proposed in the statistical (Gustafson and Hajlmarsson 1995) and NN (Moody 1991, Larsen and Hansen 1994) literature. For the comparison in section 6 , we have used a simple threshold when the authors gave no indication for the stop criterion and a validation set approximation of the risk otherwise.

\subsection{Zero Order Methods}

For linear regression models, the partial correlation coefficient can be expressed as a simple function of the weights. Although this is not sound for non linear models, there have been some attempts for using the input weight values in the computation of variable relevance. This has been observed to be an inefficient heuristic: weights cannot be easily interpreted in these models.

A more sophisticated heuristic has been proposed by Yacoub and Bennani (1997), it exploits both the weight value and the network structure of a multilayer perceptron. They derived the following criterion:

$$
S_{i}=\sum_{j \in H}\left(\frac{\left|w_{j i}\right|}{\sum_{i^{\prime} \in I}\left|w_{j i^{\prime}}\right|} \sum_{k \in O} \frac{\left|w_{k j}\right|}{\sum_{j^{\prime} \in H}\left|w_{k j^{\prime}}\right|}\right)
$$

where $I, H, O$ denote respectively the input, hidden and output layer.

For a better understanding of this measure, let us suppose that each hidden and output unit incoming weight vector has a unitary $L_{1}$ norm, the above equation can be written as:

$$
S_{i}=\sum_{o \in O j \in H}\left|w_{o j} w_{j i}\right|
$$

In (5.1.2), the inner term is the product of the weights from input $i$ to hidden unit $j$ and from $j$ to output $o$. The importance of variable $i$ for output $o$ is the sum of 
the absolute values of these products over all the paths -in the NN- from unit $i$ to unit $o$. The importance of variable $i$ is then defined as the sum of these values over all the outputs. Denominators in (5.1.1) operate as normalizing factors, this is important when using squashing functions, since these functions limit the effect of weight magnitude. Note that this measure will depend on the magnitude of the input, the different variables should then be in a similar range. The two weight layers do have different role in a MLP which is not reflected in (5.1.1), for example, if the outputs are linear, the normalization should be suppressed in the inner summation of (5.1.1).

They used a backward search and the $\mathrm{NN}$ is retrained after each variable deletion, the stop criterion is based on the evolution of the performances on a validation set, elimination is stopped as soon as performances decrease.

\subsection{First Order Methods}

Several methods propose to evaluate the relevance of a variable by the derivative of the error or of the output with respect to this variable. These evaluation criteria are easy to compute, most of them lead to very similar results. These derivatives measure the local change in the outputs wrt a given input, the other inputs being fixed. Since these derivatives are not constant like in linear models, they must be averaged over the training set. For these measures to be fully meaningful, inputs should be independent and since these measures average local sensitivity values, the training set should be representative of the input space.

\subsubsection{Saliency Based Pruning (SBP)}

This backward method (Moody and Utans 1992) uses as evaluation criterion the variation of the learning error when a variable $x_{i}$ is replaced by its empirical mean $\bar{x}_{i}$ (zero here since variables are assumed centered):

$S_{i}=\operatorname{MSE}-\operatorname{MSE}\left(\bar{x}_{i}\right)$
where $\quad \operatorname{MSE}\left(\bar{x}_{i}\right)=\frac{1}{N} \sum_{l=1}^{N}\left\|f\left(x_{1}^{l}, \ldots, \bar{x}_{i}^{l}, \ldots, x_{k}^{l}\right)-y^{l}\right\|^{2}$ 
This is a direct measure of the usefulness of the variable for computing the output. For large values of $N$, computing $S_{i}$ is costly, and a linear approximation may be used:

$$
S_{i}=\frac{1}{N} \sum_{l=1}^{N} \frac{\partial\left\|f\left(\boldsymbol{x}^{l}\right)-y^{l}\right\|^{2}}{\partial x_{i}}\left(x_{i}^{l}-\bar{x}_{i}^{l}\right)
$$

Variables are eliminated in the increasing order of $S_{i}$.

For each feature set, a NN is trained and an estimate of the generalization error - a generalization of the Final Prediction Error criterion - is computed. The model with minimum generalization error is selected.

Changes in MSE is not ambiguous only when inputs are not correlated. Variable relevance being computed once here, this method does not take into account possible correlations between variables. Relevance could be computed from the successive NNs in the sequence at a computational extra-cost $\left(\mathrm{O}\left(k^{2}\right) S_{i}\right.$ computations instead of $\mathrm{O}(k)$ in the present method).

\subsubsection{Methods using computation of output derivatives}

For a linear model the output derivative wrt any input is a constant, which is not the case for non linear NNs. Several authors have proposed to measure the sensitivity of the network transfer function with respect to input $x_{i}$ by computing the mean value of outputs derivative with respect to $x_{i}$ over the whole training set. In the case of multilayer perceptrons, this derivative can be computed progressively during learning (Hashem, 1992). Since these derivatives may take both positive and negative values, they may compensate and produce an average near zero. Most measures use average squared or absolute derivatives. Tenth of measures based on derivatives have been proposed, and many others could be defined, we thus give below only a representative sample of these measures.

The sum of the derivative absolute values has been used e.g. in Ruck et al. (1990):

$$
S_{i}=\sum_{l=1}^{N} \sum_{j=1}^{g}\left|\frac{\partial f_{j}}{\partial x_{i}}\left(x^{l}\right)\right|
$$

For classification Priddy et al. (1993) remark that since the error for decision $\mathrm{j}$ $P_{e r r}(j / \boldsymbol{x})$ may be estimated by $1-f_{j}(\boldsymbol{x}),(5.2 .3)$ may be interpreted as the 
absolute value of the error probability derivative averaged over all decisions (outputs) and data.

Squared derivatives may be used instead of the absolute values, Refenes et al. (1996) for example proposed for regression a normalized sum:

$$
S_{i}=\frac{1}{N} \frac{\operatorname{var}\left(x_{i}\right)}{\operatorname{var}(f(\boldsymbol{x})-y)} \sum_{l}\left(\frac{\partial f}{\partial x_{i}}\left(\boldsymbol{x}^{l}\right)\right)^{2}
$$

where var holds for variance. They also proposed a series of related criteria, among which:

- a normalized standard deviation of the derivatives:

$$
S_{i}=\frac{1}{N^{1 / 2}} \frac{\left(\sum_{l}\left(\frac{\partial f}{\partial x_{i}}\left(x^{l}\right)-\sum_{j} \frac{\partial f}{\partial x_{i}}\left(x^{j}\right)\right)^{2}\right)^{1 / 2}}{\sum_{l} \frac{\partial f}{\partial x_{i}}\left(x^{l}\right)}
$$

- a weighted average of the derivatives absolute values where the weights reflect the relative magnitude of $\boldsymbol{x}$ and $f(\boldsymbol{x})$ :

$$
S_{i}=\frac{1}{N} \sum_{l}\left|\frac{\partial f}{\partial x_{i}}\left(x^{l}\right) \cdot \frac{x_{i}}{f\left(x^{l}\right)}\right|
$$

All these measures being very sensitive to the input space representativeness of the sample set, several authors have proposed to use a subset of the sample in order to increase the significance of their relevance measure.

In order to obtain robust methods, "non-pathological" training examples should be discarded. For regression and radial basis function networks, Dorizzi et al. (1996) propose to use the $95 \%$ percentile of the derivative absolute value:

$$
S_{i}=q_{95}\left(\left|\frac{\partial f}{\partial x_{i}}(\boldsymbol{x})\right|\right)
$$

Aberrant points being eliminated, this contributes to the robustness of the measure. Note that the same idea could be used with other relevance measures proposed in this paper.

Following the same line, Czernichow (1996) proposed a heuristic criterion for regression, estimated on a set of non pathological examples whose cardinality is $N^{\prime}$. The proposed choice criterion is: 


$$
S_{i}=\frac{\sum_{l=1}^{N^{\prime}}\left(\frac{\partial f}{\partial x_{i}}\left(\boldsymbol{x}^{l}\right)\right)^{2}}{\max _{j}\left(\sum_{l=1}^{N^{\prime}}\left(\frac{\partial f}{\partial x j}\left(\boldsymbol{x}^{l}\right)\right)^{2}\right)}
$$

For classification, Rossi (1996), following a proposition made by Priddy et al. (1993), considers only the patterns which are near the class frontiers. He proposes the following relevance measure:

$$
S_{i}=\frac{1}{g} \sum_{\boldsymbol{x}^{l} \in \text { frontier }} \sum_{j=1}^{g} \frac{\left|\frac{\partial f_{j}}{\partial x_{i}}\left(\boldsymbol{x}^{l}\right)\right|}{\| \frac{\partial f_{j}}{\partial x}\left(\boldsymbol{x}^{l}\right) \mid}
$$

The frontier is defined as the set of point for which $\left\|\nabla_{x} f\left(x^{l}\right)\right\|>\varepsilon$ where $\varepsilon$ is a fixed threshold. Several authors have also considered relative contribution of partial derivatives to the gradient as in (5.2.9).

All these methods use a simple backward search.

For the stopping criteria, all these authors use heuristic rules, except for Refenes et al. (1996) who define statistical tests for their relevance measures. For non linear NNs, this necessitates an estimation of the relevance measure distribution, which is very costly and in our opinion usually prohibits this approach, even if it looks attractive.

\subsubsection{Links between these methods}

All these methods use simple relevance measures which depend upon the gradient of network outputs with respect to input variables. It is difficult to rank the different criteria, all that can be said is that it is wise to use some reasonable rules like discarding aberrant points for robustness, or retraining the NN after discarding each variable and computing new relevance measures for each $\mathrm{NN}$ in the sequence, in order to take into account dependencies between variables. In practice, all these methods give very similar results as will be shown in section 6 . We summarize below in table 2 the main characteristics of relevance measures for the different methods. 


\begin{tabular}{|c|c|c|c|}
\hline & $\begin{array}{c}\text { Derivative } \\
\text { used }\end{array}$ & $\begin{array}{l}\text { Task } \\
\text { C/R }\end{array}$ & $\begin{array}{l}\text { Data } \\
\text { used }\end{array}$ \\
\hline (Moody (5.2.1)) & $\frac{\partial f}{\partial x_{i}}$ & $\mathrm{C} / \mathrm{R}$ & All \\
\hline (Refenes (5.2.5)) & $\frac{\partial f}{\partial x_{i}}$ & $\mathrm{C} / \mathrm{R}$ & All \\
\hline (Dorizzi (5.2.7)) & $\left|\frac{\partial f}{\partial x_{i}}\right|$ & $\mathrm{C} / \mathrm{R}$ & $\begin{array}{c}\text { Non pathological } \\
\text { data }\end{array}$ \\
\hline (Refenes (5.2.6)) & $\left|\frac{\partial f}{\partial x_{i}}\right|$ & $\mathrm{C} / \mathrm{R}$ & All \\
\hline (Czernichow (5.2.8)) & $\left(\frac{\partial f}{\partial x_{i}}\right)^{2}$ & $\mathrm{C} / \mathrm{R}$ & $\begin{array}{c}\text { Non pathological } \\
\text { data }\end{array}$ \\
\hline (Refenes (5.2.4)) & $\left(\frac{\partial f}{\partial x_{i}}\right)^{2}$ & $\mathrm{C} / \mathrm{R}$ & All \\
\hline (Ruck (5.2.3)) & $\sum_{j=1}^{g}\left|\frac{\partial f_{j}}{\partial x_{i}}\right|$ & $\mathrm{C}$ & All \\
\hline$(\operatorname{Rossi}(5.2 .9))$ & $\sum_{j=1}^{g}\left|\frac{\partial f_{j}}{\partial x_{i}}\right| / \| \nabla_{x} f_{j} \mid$ & $\mathrm{C}$ & $\begin{array}{c}\text { Frontier between } \\
\text { classes }\end{array}$ \\
\hline
\end{tabular}

Table 2. Computation of the relevance of a variable by different methods using the derivative of the network function. $C / R$ denote respectively Classification and Regression tasks.

\subsection{Second Order Methods}

Several methods propose to evaluate the relevance of a variable by computing weight pruning criteria for the set of weights of each input node. We present below three methods. The first one is a Bayesian approach for computing the weight variance. The other two use the hessian of the cost function for computing the cost function dependence upon input unit weights.

\subsubsection{Automatic Relevance Determination (ARD)}

This method was proposed by MacKay (1994) in the framework of Bayesian learning. In this approach, weight are considered as random variables and 
regularization terms taking into account each input are included into the cost function. Assuming that the prior probability distribution of the group of weights for the $i$ th input is gaussian, the input posterior variance $\sigma_{i}^{2}$ is estimated (with the help of the hessian matrix).

ARD has been successful for time serie prediction, learning with regularization terms improved the prediction performances. However ARD has not really been used as a feature selection method since variables were not pruned during training.

\subsubsection{Optimal Cell Damage}

Several neural selection methods have been inspired by weight pruning techniques. For the latter, the decision of pruning a weight is made according to a relevance criterion often named the weight saliency, the weight being pruned if its saliency is low. Similarly, the saliency for an input cell is usually defined as the sum of its weights saliencies.

$$
\operatorname{Saliency}\left(x_{i}\right)=\sum_{\text {fan-out }(i)} \operatorname{Saliency}\left(w_{j}\right)
$$

where fan-out $(i)$ is the set of weights of input $i$.

Optimal Cell Damage (OCD) has been proposed by Cibas et al. (1994a, 1996) (A similar method has also been proposed by Mao et al., 1994). This feature selection method is inspired from the Optimal Brain Damage (OBD) weight pruning technique developed by LeCun (1990). In OBD, the connection saliency is defined by :

$$
\operatorname{Saliency}\left(w_{j}\right)=\frac{1}{2} H_{j j} w_{j}^{2}=\frac{1}{2} \frac{\partial^{2} M S E}{\partial w_{j}^{2}} w_{j}^{2}
$$

which is an order two Taylor expansion of MSE variation around a local minimum. The Hessian matrix $H$ can be easily computed using gradient descent but this may be computationally intensive for large networks. For OBD, the authors use a diagonal approximation for the hessian which can then be computed in $O(N)$. The saliency of an input variable is defined accordingly as:

$$
S_{i}=\operatorname{Saliency}\left(x_{i}\right)=\frac{1}{2} \sum_{j \in \text { fan-oux }(i)} \frac{\partial^{2} M S E}{\partial w_{j}^{2}} w_{j}^{2}
$$

Cibas et al. (1994) proposed to use (5.3.5) as a choice criterion for eliminating variables. The NN is trained so as to reach a local minimum, variables whose 
saliency is below a given threshold are eliminated. The threshold value is fixed by cross validation. This process is then repeated until no variable is found below the threshold.

This method has been tested on several problems and gave satisfying results. Once again, the difficulty lies in selecting an adequate threshold. Furthermore, since several variables can be eliminated simultaneously whereas only individual variable pertinence measures are used, significant sets of dependent variables may be eliminated.

For stopping, the generalization performances of the NN sequence are estimated via a validation set and the variable set corresponding to the $\mathrm{NN}$ with the best performances is chosen.

The hessian diagonal approximation has been questioned by several authors, Hassibi and Stork (1993), for example, proposed a weight pruning algorithm, Optimal Brain Surgeon (OBS) which is similar to OBD, but uses the whole hessian for computing weight saliencies. Stahlberger and Riedmiller (1997) proposed a feature selection method similar to OCD except that it takes into account non diagonal terms in the hessian.

For all these methods, saliency is computed using for performance measure the error variation on the training set. Weight estimation and model selection both use the same data set, which is not optimal. Pedersen et al. (1996) propose two weight pruning methods $\gamma \mathrm{OBD}$ and $\gamma \mathrm{OBS}$ that compute weight saliency according to an estimate of the generalization error: the Final Prediction Error (Akaike 1970). Similarly to OBD and OBS, these methods could be also transformed into feature selection methods.

\subsubsection{Early Cell Damage (ECD)}

Using a second order Taylor expansion, as in the OBD family of methods, is justified only when a local minimum is reached and the cost is locally quadratic in this minimum. Both hypothesis are barely met in practice. Tresp et al. (1997) propose two weight pruning techniques from the same family, coined EBD (Early Brain Damage) and EBS (Early Brain Surgeon). They use a heuristic justification to take into account early stopping by adding a new term in the saliency computation. 
These methods can be extended for feature ranking, we will call ECD (Early Cell Damage) the EBD extension. For ECD, the saliency of input $i$ is defined as:

$$
S_{i}=\sum_{j \in \text { fan-out }(i)} \frac{1}{2} \frac{\partial^{2} M S E}{\partial w_{j}^{2}} w_{j}^{2}-\frac{\partial M S E}{\partial w_{j}} w_{j}+\frac{1}{2} \frac{\left(\frac{\partial M S E}{\partial w_{j}}\right)^{2}}{\frac{\partial^{2} M S E}{\partial w_{j}^{2}}}
$$

The algorithm we propose is slightly different from OCD: only one variable is eliminated at a time, and the $\mathrm{NN}$ is retrained after each deletion.

For choosing the "best" set of variables, we have used a variation of the "selection according to an estimate of the generalization error" method. This estimate is computed using a validation set. Since the performances may oscillate and be not significantly different, several subsets may have the same performances (see e.g. figure 1). Using a Fisher test we compare any model performances with those of the best model, we then select the set of networks whose performances are similar to the best ones and choose among these networks the one with the smallest number of input variables.

\section{Experimental comparison}

We now present comparative performances of different feature selection methods. Comparing these methods is a difficult task: there is not a unique measure which characterizes the importance of each input, the selection accuracy also depends on the search technique and on the variable subset choice criterion. In the case of NNs, these different steps rely on heuristics which could be exchanged from one method to the other. The NNs used are multilayer perceptrons with one hidden layer of 10 neurons.

The comparison we provide here is not intended for a definite ranking of the different methods but for illustrating the general behavior of some of the methods which have been described before. We have used two synthetic classification problems which illustrate different difficulties of variable selection. In the first one the frontiers are "nearly" linear and there are dependent variables as well as pure noise variables. The second problem has non linear frontiers and variables can be chosen independent or correlated. 
The first problem has been originally proposed by Breiman et al. (1984). It is a three class waveforms classification problem with 19 noisy dependent features. We have also used a variation of this problem where 21 pure noise variables are added to the 19 initial variables (there are 40 inputs for this variant). The training set has 300 patterns and the test set 4300 . A description of this problem is provided in the appendix. The performances of the optimal Bayes classifier estimated on this test set are $86 \%$ correct classification. A performance comparison appears in tables 3 and 4 for these two instances.

\begin{tabular}{|c|c|c|c|}
\hline Method & $\mathbf{p}^{*}$ & Selected Variables & Perf. \\
\hline None & 40 & 111111111111111111111111111111111111111 & $\begin{array}{c}82.51 \% \\
{[81.35-83.62]}\end{array}$ \\
\hline Stepdisc (4.2.2) & 14 & 0001101111111110111000000000000000000000 & $\begin{array}{c}85.35 \% \\
{[84.26-86.38]}\end{array}$ \\
\hline (Bonnlander (4.3.2)) & 12 & 0000111011111111100000000000000000000000 & $\begin{array}{c}85.12 \% \\
{[84.02-86.15]}\end{array}$ \\
\hline (Yacoub (5.1.1)) & 16 & 0001111111111111111000000000000000000000 & $\begin{array}{c}85.16 \% \\
{[84.07-86.19]}\end{array}$ \\
\hline (Moody (5.2.1)) & 16 & 0001111111111111111000000000000000000000 & $\begin{array}{c}85.19 \% \\
{[84.10-86.22]}\end{array}$ \\
\hline $\begin{array}{c}\text { (Ruck (5.2.3)) } \\
(\text { Dorizzi (5.2.7)) }\end{array}$ & 18 & 0111111111111111111000000000000000000000 & $\begin{array}{c}85.51 \% \\
{[84.43-86.53]}\end{array}$ \\
\hline (Czernichow (5.2.8)) & 17 & 0101111111111111111000000000000000000000 & $\begin{array}{c}85.67 \% \\
{[84.59-86.69]}\end{array}$ \\
\hline$($ Cibas $(5.3 .5))$ & 9 & 0000011111101110000000000000000000000000 & $\begin{array}{c}82.26 \% \\
{[81.09-83.37]}\end{array}$ \\
\hline (Leray (5.3.6)) & 11 & 0000011111111111000000000000000000000000 & $\begin{array}{c}84.56 \% \\
{[83.45-85.61]}\end{array}$ \\
\hline
\end{tabular}

Table 3. Performance comparison of different variable selection on the noisy wave problem.

For the noisy problem, all methods do eliminate pure noise variables. Except for the two methods at the bottom of table 3 which give slightly lower performances and select fewer variables, all give similar values around $85 \%$ correct. Stepdisc also gives good performances since in this problem data have a unimodal distribution and the frontiers are nearly linear. For the non noisy problem, performances and methods ordering change. The two techniques at the bottom of table 4 give now slightly better performances. 


\begin{tabular}{|cccc|}
\hline \hline Method & p $^{*}$ & Selected Variables & Perf. \\
\hline \hline None & 21 & 111111111111111111111 & $\begin{array}{c}85.28 \% \\
{[84.19-86.31]}\end{array}$ \\
\hline \hline Stepdisc (4.2.2) & 14 & 001110101111111011100 & $\begin{array}{c}84.19 \% \\
{[83.07-85.25]}\end{array}$ \\
\hline (Bonnlander (4.3.2)) & 8 & 000001100111101010000 & $\begin{array}{c}83.05 \% \\
{[81.90-84.14]}\end{array}$ \\
\hline \hline$($ Yacoub (5.1.1)) & 18 & 011111111111111111100 & $\begin{array}{c}85.46 \% \\
{[84.38-86.48]}\end{array}$ \\
\hline (Moody (5.2.1)) & 16 & 000111111111111111100 & $\begin{array}{c}85.63 \% \\
{[84.65-86.65]}\end{array}$ \\
\hline $\begin{array}{c}\text { (Ruck (5.2.3)) } \\
\text { (Dorizzi (5.2.7)) }\end{array}$ & 12 & 000111101111111010000 & $\begin{array}{c}84.65 \% \\
{[83.54-85.70]}\end{array}$ \\
\hline$($ Czernichow (5.2.8)) & 10 & 000110101011111010000 & $\begin{array}{c}82.58 \% \\
{[81.42-83.68]}\end{array}$ \\
\hline \hline$($ Cibas (5.3.5)) & 15 & 001011111111111110100 & $\begin{array}{c}85.23 \% \\
{[84.14-86.26]}\end{array}$ \\
\hline (Leray (5.3.6)) & 13 & 000011111111111110000 & $\begin{array}{c}85.67 \% \\
{[84.59-86.69]}\end{array}$ \\
\hline \hline
\end{tabular}

Table 4. Performance comparison of different variable selection methods on the original wave problem.

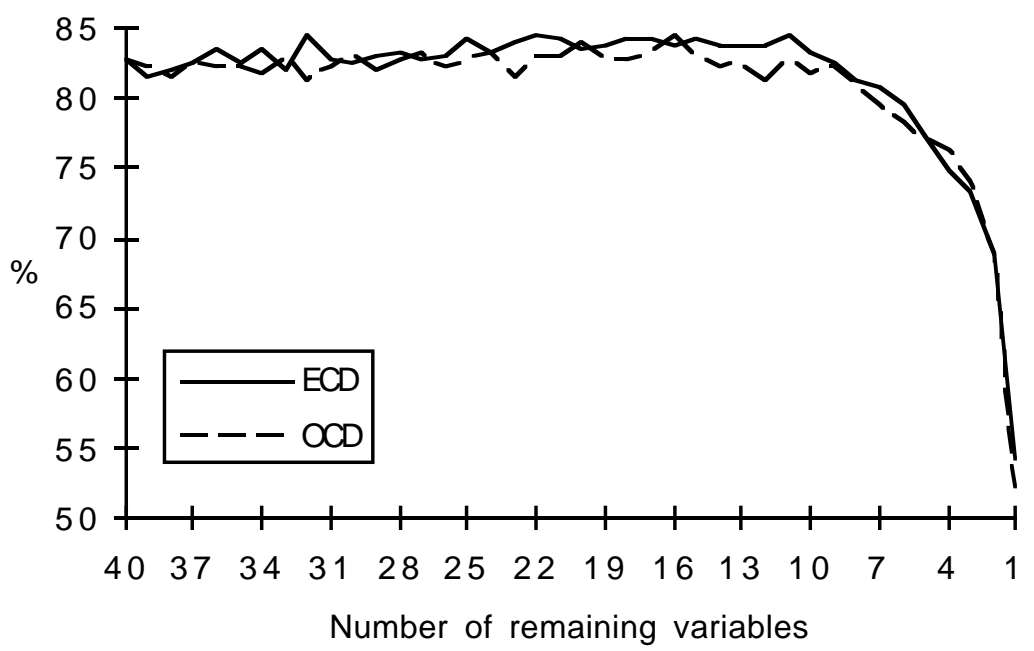

Figure 1. Performance comparison of two variable selection methods (OCD and $E C D)$ according to the number of remaining variables for the noisy wave problem.

Figure 1 shows performance curves for two methods, OCD and ECD, estimated on a validation set. Since we have used a single validation set, there are small 
fluctuations in the performances. Some form of cross validation should be used in order to get better estimates, the test strategy proposed for ECD looks also attractive in this case. It can be seen that for this problem, performances are more or less similar during the backward elimination (they slightly rise) until they quickly drop when relevant variables are removed.

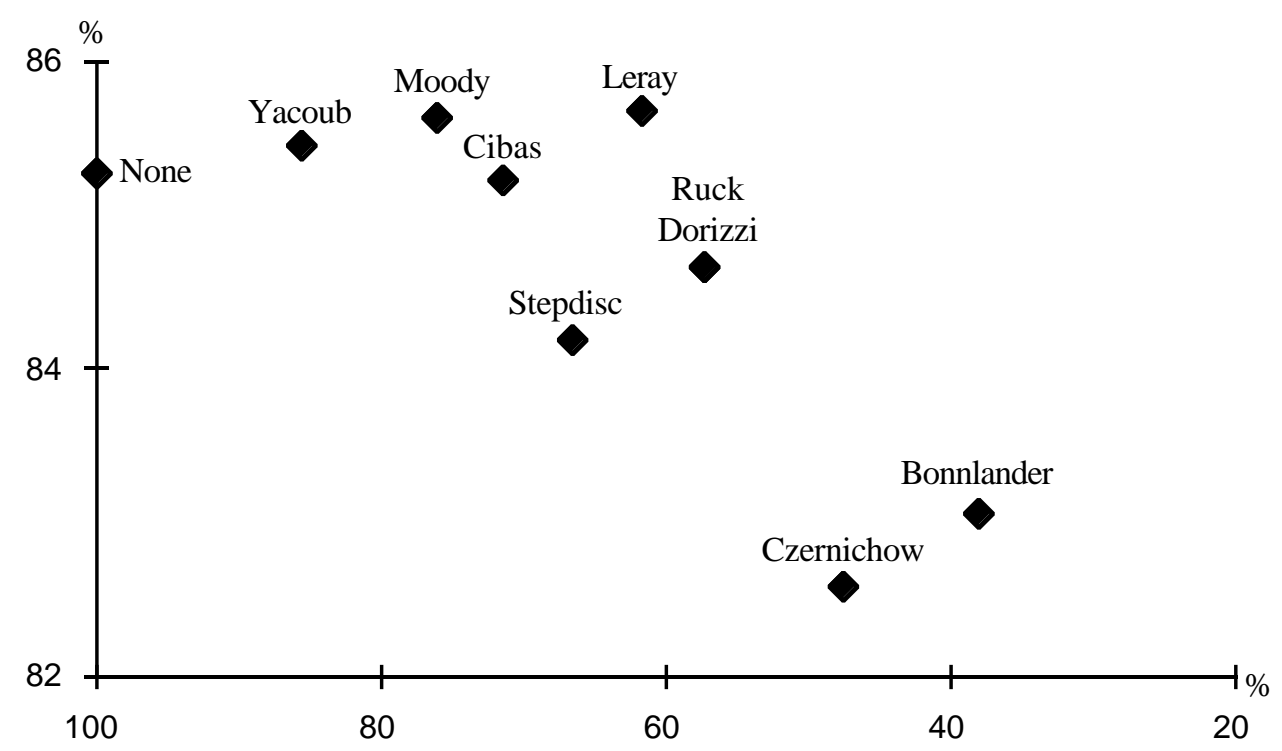

Figure 2. Performance comparison of different variable selection methods vs. percentage of selected variables on the original wave problem. $x$ axis: percentage of variables selected, y axis: percentage of correct classification.

Figure 2 gives the repartition of different variable selection methods for the original wave problem according to their performances (y axis) and the percentage of selected variables (x axis). The best methods are those with the best performances and the lower number of variables. In this problem, "Leray" is satisfying (see figure 2). "Yacoub" does not delete enough variables while "Bonnlander" deletes too much variables.

The second problem is a two class problem in a 20 dimensional space. The classes are distributed according two gaussians with respectively $\mu_{l}=(0, \ldots, 0)$, $\Sigma_{1}=4 * I, \mu_{2}=(0,1,2, \ldots, 19) / \alpha\left(\alpha\right.$ is chosen so that $\left.\left\|\mu_{1} \mu_{2}\right\|=2\right)$ and $\Sigma_{2}=I$. In this problem, variable relevance is ordered according to their index: $x_{1}$ is useless, $x_{i+1}$ is more relevant than $x_{i}$. 


\begin{tabular}{|cccc|}
\hline \hline Method & p $^{*}$ & Selected Variables & Perf. \\
\hline \hline None & 20 & 11111111111111111111 & $\begin{array}{c}94.80 \% \\
{[94.15-94.35]}\end{array}$ \\
\hline \hline Stepdisc (4.2.2) & 17 & 10001111111111111111 & $\begin{array}{c}94.88 \% \\
{[94.23-95.43]}\end{array}$ \\
\hline (Bonnlander (4.3.2)) & 5 & 00010000000000011011 & $\begin{array}{c}90.60 \% \\
{[89.76-91.38]}\end{array}$ \\
\hline \hline (Yacoub (5.1.1)) & 18 & 01011111111111111111 & $\begin{array}{c}94.86 \% \\
{[94.21-95.44]}\end{array}$ \\
\hline (Moody (5.2.1)) & 9 & 01000100011000110111 & $\begin{array}{c}92.94 \% \\
{[92.20-93.62]}\end{array}$ \\
\hline (Ruck (5.2.3)) & 10 & 00000000101101111111 & $\begin{array}{c}94.86 \% \\
{[94.21-95.44]}\end{array}$ \\
\hline (Dorizzi (5.2.7)) & 11 & 00000000101111111111 & $\begin{array}{c}94.66 \% \\
{[94.00-95.25]}\end{array}$ \\
\hline$($ Czernichow (5.2.8)) & 9 & 00000000001101111111 & $\begin{array}{c}94.02 \% \\
{[93.33-94.02]}\end{array}$ \\
\hline \hline (Cibas (5.3.5)) & 14 & 01001110010111111111 & $\begin{array}{c}94.62 \% \\
{[93.96-95.21]}\end{array}$ \\
\hline (Leray (5.3.6)) & 15 & 01011011101110111111 & $\begin{array}{c}94.08 \% \\
{[93.39-94.70]}\end{array}$ \\
\hline \hline
\end{tabular}

Table 5. Performance comparison of different variable selection methods on the two gaussian problem with uncorrelated variables.

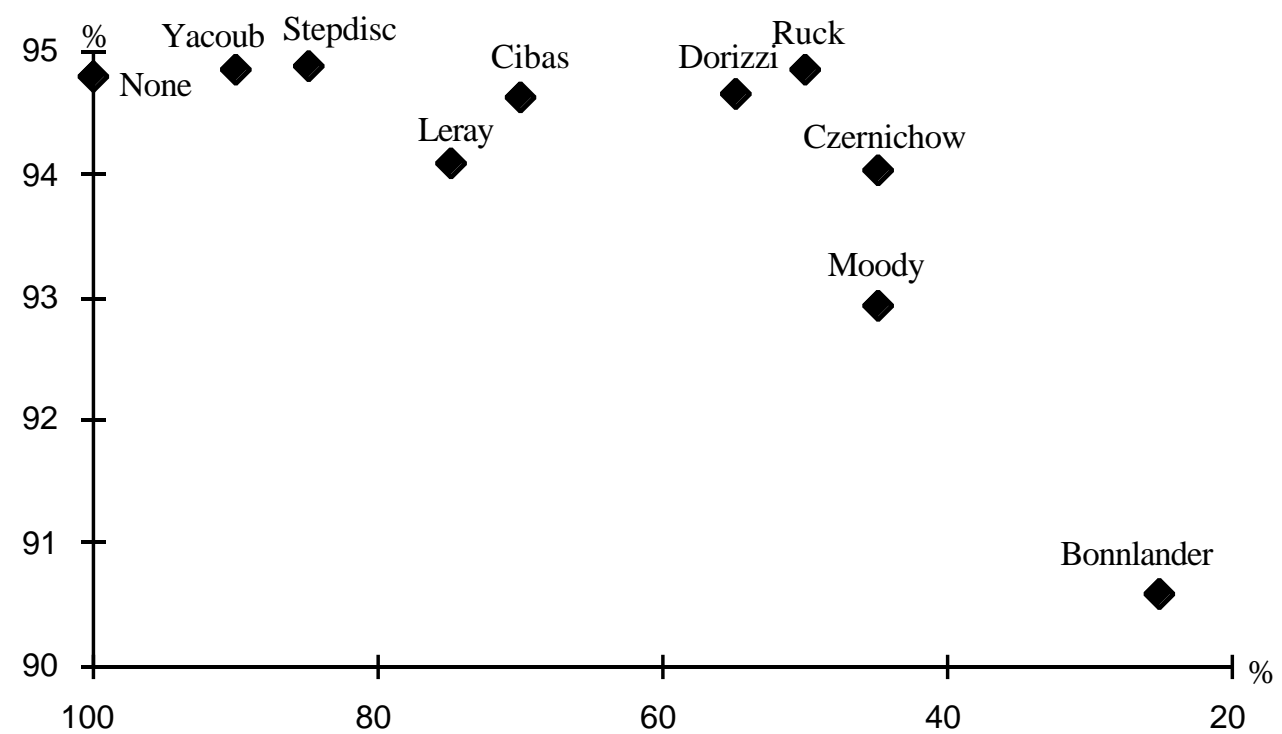

Figure 3. Performance comparison of different variable selection methods vs. percentage of selected variables on the two gaussian problem with uncorrelated variables. $x$ axis: percentage of variables selected, y axis: percentage of correct classification. 
Table 5 shows that Stepdisc is not adapted for this non linear frontier: it is the only method that selects $x_{1}$ which is useless for this problem. We can remark on figure 3 that Bonnlander's method deletes too many variables whereas Yacoub's stop criterion is too rough and does not delete enough variables.

In an other experiment, we replaced the $I$ matrix in $\Sigma_{1}$ and $\Sigma_{2}$ by a block diagonal matrix. Each block is $5 \times 5$ so that there are four groups of five successive correlated variables in the new problem.

\begin{tabular}{|cccc|}
\hline \hline Method & $\mathbf{p}^{*}$ & Selected Variables & Perf. \\
\hline \hline None & 20 & 111111111111111111111 & $\begin{array}{c}90.58 \% \\
{[89.74-91.36]}\end{array}$ \\
\hline \hline Stepdisc (4.2.2) & 11 & 00001101011010110111 & $\begin{array}{c}91.96 \% \\
{[91.17-92.68]}\end{array}$ \\
\hline (Bonnlander (4.3.2)) & 5 & 00001001010000100001 & $\begin{array}{c}88.48 \% \\
{[85.57-89.34]}\end{array}$ \\
\hline \hline (Ruck (5.2.3)) & 10 & 00011001011110100011 & $\begin{array}{c}91.06 \% \\
{[90.24-91.82]}\end{array}$ \\
\hline \hline (Leray (5.3.6)) & 7 & 00000010101010100011 & $\begin{array}{c}90.72 \% \\
{[89.88-91.49]}\end{array}$ \\
\hline \hline
\end{tabular}

Table 6. Performance comparison of different variable selection methods on the two gaussian problem with correlated variables.

Table 6 gives the results of some representative methods for this problem:

- Stepdisc still gives a model with good performances but selects many correlated variables,

- Bonnlander's method selects only 5 variables and gives significantly lower results,

- Ruck's method obtains good performances but selects some correlated variables,

- Leray's method, thanks to the retraining after each variable deletion, find models with good performances and few variables (7 compared to 10 and 11 for Ruck and Stepdisc). 


\section{Conclusion}

We have reviewed variable selection methods developed in the field of Neural Networks. The main difficulty here is that NNs are non linear systems which do not make use of explicit parametric hypothesis. As a consequence, selection methods rely heavily on heuristics for the three steps of variable selection : relevance criterion, search procedure - NN variable selection use mainly backward search - and choice of the final model. We first discussed the main difficulties for developing each of these steps. We then introduced different families of methods and discussed their strengths and weaknesses. We believe that a variable selection method must remain computationally feasible for being useful, and we have not considered techniques which rely on computer intensive methods like e.g. bootstrap at each step of the selection . Instead, we have proposed a series of rules which could be used in order to enhance several of the methods which have been described, at a reasonable extra computational cost, e.g. retraining each $\mathrm{NN}$ in the sequence and computing the relevance for each of these NN allows to take into account some correlations between variables, simple estimates of the generalization error may be used for the evaluation of a variable subset, simple tests on these estimates, allow to choose minimal variable sets (section 5.3.3). Finally we performed a comparison of representative NN selection techniques on synthetic problems.

\section{References}

Akaike, H. (1970). Statistical Predictor Identification, Ann. Inst. Statist. Math. 22:203-217.

Battiti, R. (1994). Using Mutual Information for Selecting Features in Supervised Neural Net Learning, IEEE Transactions on Neural Networks 5(4):537-550.

Baxt, W.G. and White, H. (1995). Bootstrapping confidence intervals for clinical input variable effects in a network trained to identify the presence of acute myocardial infraction, Neural Computation 7:624-638.

Bonnlander, B.V. and Weigend, A.S. (1994). Selecting Input Variables Using Mutual Information and Nonparametric Density Evaluation, in Proceedings of ISANN'94. 42-50.

Breiman, L., Friedman, J., Olshen R., and Stone, C. (1984). Classification and Regression Trees. Wadsworth International Group.

Cibas, T. Fogelman Soulié, F. Gallinari, P. and Raudys, S. (1994a). Variable Selection with Optimal Cell Damage. In Proceedings of ICANN'94.

Cibas, T. Fogelman Soulié, F. Gallinari, P. and Raudys, S. (1996). Variable Selection with Neural Networks. Neurocomputing 12:223-248.

Czernichow, T. (1996). Architecture Selection through Statistical Sensitivity Analysis. In Proceedings of ICANN'96, Bochum, Germany. 
Dorizzi, B. Pellieux, G. Jacquet, F Czernichow, T. and Munoz, A. (1996). Variable Selection Using Generalized RBF Networks : Application to the Forecast of the French T-Bonds. In Proceedings of IEEE-IMACS'96, Lille, France.

Fraser, A.M. and Swinney, H.L. (1986). Independent Coordinates for Strange Attractors from Mutual Information, Physical Review A:33(2):1134-1140.

Goutte, C. (1997). Extracting the Relevant Decays in Time Series Modelling, Neural Networks for Signal Processing VII, Proceedings of the IEEE Workshop, Neural Networks for Signal Processing VII, Proceedings of the IEEE Workshop.

Gustafson and Hajlmarsson (1995). 21 maximum likelihood estimators for model selection. Automatica.

Habbema, J.D.F and Hermans, J. (1977). Selection of Variables in Discriminant Analysis by F-statistic and Error Rate, Technometrics, 19(4):487-493.

Härdle, W. (1990). Applied Nonparametric Regression. Cambridge University Press. Econometric Society Monograph n.19.

Hashem, S. (1992). Sensitivity Analysis for Feedforward Artificial Neural Networks with Differentiable Activation Functions. In Proceedings of 1992 International Joint Conference on Neural Networks IJCNN92 I:419-424.

Hassibi, B. and Stork, D.G. (1993). Second Order Derivatives for Network Pruning : Optimal Brain Surgeon. Neural Information Processing Systems 5:164-171.

Kittler, J. (1986). Feature Selection and Extraction, Chapter 3 in Handbook of Pattern Recognition and Image Processing, Eds. Tzay Y. Young, King-Sun Fu, Academic Press. 59-83.

Larsen, J. and Hansen, L.K. (1994). Generalized performances of regularized neural networks models. Proceedings of the 1994 IEEE Workshop on Neural Networks for Signal Processing. 42-51.

LeCun, Y. Denker, J.S. and Solla, S.A. (1990). Optimal Brain Damage. Neural Information Processing Systems 2:598-605.

MacKay, D.J.C. (1994). Bayesian Non-linear Modelling for the Energy Prediction Competition. In ASHRAE Transactions. 1053-1062.

Mao, J. Mohiuddin, K. and Jain, A.K. (1994). Parsimonious Network Design and Feature Selection Through Node Pruning. In Proceedings of the 12th International Conference on Pattern Recognition. 622-624.

McLachlan, G.J. (1992). Discriminant Analysis and Statistical Pattern Recognition, WileyInterscience publication.

Moody, J. (1991). Note on generlization, regularization and architecture selection in non linear learning systems. Proceedings of the first IEEE Workshop on Neural Networks for Signal Processing. 1-10.

Moody, J. and Utans, J. (1992). Principled Architecture Selection for Neural Networks: Application to Corporate Bond Rating Prediction. Neural Information Processing Systems 4.

Moody, J. (1994). Prediction Risk and Architecture Selection for Neural Networks in From Statistics to Neural Networks - Theory and Pattern Recognition Applications, Eds V. Cherkassky, J.H. Friedman, H. Wechsler, Springer-Verlag.

Narendra, P.M. and Fukunaga, K. (1977). A Branch and Bound Algorithm for Feature Subset Selection. IEEE Transactions on Computers 26(9):917-922.

Pedersen, M.W. Hansen, L.K. and Larsen, J. (1996). Pruning with generalisation based weight saliencies: $\gamma O B D, \gamma O B S$. Neural Information Processing Systems 8.

Priddy, K.L. Rogers, S.K. Ruck, D.W. Tarr, G.L. and Kabrisky, M. (1993). Bayesian Selection of Important Features for Feedforward Neural Networks. Neurocomputing 5:91103. Elsevier ed.

Pudil, P., Novovicova, J. and Kittler, J. (1994). Floating search methods in feature selection. Pattern Recognition Letters 15:1119-1125. 
Refenes, A.N. Zapranis, A. and Utans J. (1996). Neural Model Identification, Variable Selection and Model Adequacy. In Neural Networks in Financial Engineering, Proceedings of NnCM-96.

Rossi, F. (1996). Attribute Suppression with Multi-Layer Perceptron. In Proceedings of IEEEIMACS'96, Lille, France.

Ruck, D.W. Rogers, S.K. and Kabrisky, M. (1990). Feature Selection Using a MultiLayer Perceptron. In J. Neural Network Comput. 2 (2):40-48.

Stahlberger, A. and Riedmiller, M. (1997). Fast Network Pruning and Feature Extraction Using the Unit-OBS Algorithm. Neural Information Processing Systems 9:655-661.

Thompson M.L. (1978). Selection of Variables in Multiple Regression. Part I: A Review and Evaluation, International Statistical Review, 46:1-19, Selection of Variables in Multiple Regression. Part II: Chosen Procedures, Computations and Examples, in International Statistical Review, 46:129-146.

Tresp, V. Neuneier, R. and Zimmermann, G. (1997). Early Brain Damage. Neural Information Processing Systems 9:669-675.

Van de Laar, P. Gielen, S. and Heskes, T. (1997). Input Selection with Partial Retraining. In Proceedings of ICANN'97.

White, H. (1989). Learning in Artificial Neural Networks : A Statistical Perspective. Neural Computation 1:425-464.

Wilks, S.S. (1963). Mathematical Statistics, Wiley, New York.

Yacoub, M. and Bennani, Y. (1997). HVS: A Heuristic for Variable Selection in Multilayer Artificial Neural Network Classifier. in Proceedings of ANNIE'97. 527-532.

\section{Appendix : waveforms problem}

This problem has been proposed by Breiman et al. (1984). 3 vectors or waveforms in 21 dimensions, $H^{i}, i=1, \ldots, 3$, are given. Patterns in each class are defined in $\Re^{21}$ as random convex combinations of 2 of these vectors (waves $(1,2),(1,3),(2,3)$ respectively for class 1,2 and 3$)$.

The problem is then to classify these patterns into one of the 3 classes. More precisely, patterns are generated according to:

$$
x_{i}=\frac{u H_{i}^{m}+(1-u) H_{i}^{n}}{5}+\varepsilon_{i}
$$$$
0 \leq i \leq 20
$$

where $x_{i}$ denotes the $i^{\text {th }}$ component of a pattern $\boldsymbol{x}, u$ is a uniform random variable in $[0,1], \varepsilon_{i}$ is generated according to a normal distribution $\mathrm{N}(0,1), m$ and $n$ identify the two waves used in this combination, i.e. the class of pattern $\boldsymbol{x}$.

For the noisy problem, 19 additional components are added to the 21 components of the above vectors:

$$
x_{i}=\varepsilon_{i} \quad 21 \leq i \leq 40
$$

The training, validation and test sets have respectively 300, 1000, 4300 elements. 